\title{
Radiation Characteristics of Glass Containing Gas Bubbles
}

\author{
Laurent Pilon* \\ Mechanical and Aerospace Engineering Department, Henri Samueli School of Engineering and Applied Science, \\ University of California, Los Angeles, California 90095
}

Raymond Viskanta

School of Mechanical Engineering, Purdue University, West Lafayette, Indiana 47907

\begin{abstract}
In many materials processing and manufacturing situations such as steel, aluminum, ceramics, and glass, gas bubbles can form in liquid and solid phases. The presence of such bubbles affects the thermophysical properties and radiation characteristics of the two-phase system and hence the transport phenomena. This paper presents a general formulation of the radiation characteristics of semitransparent media containing large gas bubbles (bubble radius is much larger than the wavelength of radiation). Sample calculations for the spectral absorption and extinction coefficients and single scattering albedo of soda-lime silicate glass containing bubbles are discussed. Particular attention is paid to the effect of the volumetric void fraction and the bubble size distribution. Results clearly show that the presence of bubbles strongly affects the radiation characteristics of the semitransparent media containing entrapped gas bubbles, particularly if bubbles, void fractions, and the spectral absorption coefficient of the continuous phase are small.
\end{abstract}

\section{Introduction}

I $\mathrm{N}$ MANY materials processing and manufacturing situations such as steel, aluminum, ceramics, and glass, gas bubbles can form in liquid and solid phases. The presence of such bubbles affects the thermophysical properties and radiation characteristics of the two-phase system and hence the transport phenomena. In glassmelting furnaces, for example, a large number of bubbles are formed by chemical reactions during melting of the batch and the thermal decomposition of refining agents. Bubbles that are large enough rise at the surface of the glass melt while small bubbles are trapped in the flowing molten glass. The quality of glass products is degraded if gas bubbles and unfused silica grains remain in the molten glass as it is being pulled from the furnace. ${ }^{2,3}$ The quality of the glass produced is significantly affected by the flow pattern and the temperature of the glass melt. Both depend strongly on the heat transfer from the combustion space to the batch and to the molten glass. The high temperatures and the oxidizing environment in the furnace make experimental measurements very difficult and unreliable. Therefore, numerical simulations constitute appropriate alternatives for better design and control of the glass-melting furnaces. General models for predicting the flow and the temperature fields have been reviewed by Viskanta. ${ }^{14}$ All the models use the Rosseland diffusion approximation for radiative heat transfer in the molten glass. Recently, Cheong et al. ${ }^{4}$

J. R. Hellmann—contributing editor

Manuscript No. 187364. Received October 30, 2001; approved August 20, 2002.

This work was supported by the U.S. Department of Energy/Glass Industry/ Argonne National Laboratory/University collaborative research project. questioned this assumption and showed that the diffusion approximation for radiative transfer is not recommended when the depth of the glass layer is less than $0.5 \mathrm{~m}$. Instead, they suggested the use of the P-1 approximation not only for shallow glass melt layers but also for deep ones.

The analyses reported in the literature have neglected the presence of numerous gas bubbles of different sizes in the melt that may affect the radiation heat transfer. The presence of the bubbles will affect the radiation characteristics of the glass melt since the radiation is scattered by the bubbles. Approximate and rigorous treatements of radiative transfer in glass require spectral radiation characteristics of glass melt which contains gas bubbles. Therefore, it is of particular interest to understand the effect of bubbles on the radiation characteristics of the molten glass in order to better predict radiation heat transfer in glass-melting furnaces, and improve the glass quality and the energy efficiency of the processes. The objective of this work is to assess the effect of the bubble radius, the bubble size distribution, and the void fraction on the radiation characteristics of glass containing spherical gas bubbles with different bubble size distributions and to gain understanding of their importance on radiative transfer in glass.

\section{Analysis}

Consider heat transfer within a horizontal layer of continuous condensed phase containing bubbles as shown in Fig. 1. In general, convection (due to the motion of bubbles), conduction, and radiation heat transfer are present and should be considered. We further assume that the continuous condensed phase is a solid or a slowly moving liquid and is essentially isothermal. Then, the first two modes of heat transfer can be safely neglected in comparison to heat transfer by radiation. When gas bubbles are moving with the liquid phase, a time-averaged void fraction and bubble size distribution should be used. In addition, we assume the following: (1) all bubbles are spherical, (2) the scattering of a single bubble is not affected by the presence of its neighbors (independent scattering); and (3) the radiation field within the liquid layer is incoherent (i.e., scattering centers are randomly distributed with zero-phase correlation). Then, radiative transfer within an absorbing, emitting, and independently scattering medium is governed by the integrodifferential equation expressed in dimensionless optical coordinates: ${ }^{10,15}$

$$
\begin{aligned}
\frac{\mathrm{d} I_{\lambda}}{d \tau_{\lambda}}= & {\left[1-\omega_{\lambda}(\hat{s})\right] I_{\mathrm{b} \lambda}[T(\hat{s})]-I_{\lambda}(\hat{s}, \hat{\Omega}) } \\
& +\frac{\omega_{\lambda}(\hat{s})}{4 \pi} \int_{\hat{\Omega}^{\prime}=4 \pi} I_{\lambda}\left(\hat{s}, \hat{\Omega}^{\prime}\right) \Phi_{\lambda}\left(\hat{s}, \hat{\Omega}^{\prime} \rightarrow \hat{\Omega}\right) \mathrm{d} \hat{\Omega}^{\prime}
\end{aligned}
$$

Here, $\hat{s}$ is the local spatial coordinate unit vector, $\hat{\Omega}$ is a line-of-sight direction, and $I_{\mathrm{b} \lambda}$ is Planck's blackbody function. The 
spectral optical thickness $\tau_{\lambda}$ and single scattering albedo $\omega_{\lambda}$ are defined, respectively, as

$$
\begin{aligned}
& \tau_{\lambda}=\int_{0}^{s}\left(\kappa_{\lambda}+\sigma_{\lambda}\right) \mathrm{d} s=\int_{0}^{s} \beta_{\lambda} \mathrm{d} s \\
& \omega_{\lambda}=\frac{\sigma_{\lambda}}{\kappa_{\lambda}+\sigma_{\lambda}}=\frac{\sigma_{\lambda}}{\beta_{\lambda}}
\end{aligned}
$$

where $\kappa_{\lambda}, \sigma_{\lambda}$, and $\beta_{\lambda}\left(=\kappa_{\lambda}+\sigma_{\lambda}\right)$ are the absorption, scattering, and extinction coefficients, respectively. The scattering phase function $\Phi_{\lambda}\left(\hat{s}, \hat{\Omega}^{\prime} \rightarrow \hat{\Omega}\right)$ represents the probability of scattering of the radiation from a beam propagating in the direction $\hat{\Omega}^{\prime}$ to the direction $\hat{\Omega}$, and it is normalized such that

$$
\frac{1}{4 \pi} \int_{\hat{\Omega}^{\prime}=4 \pi} \Phi_{\lambda}\left(\hat{s}, \hat{\Omega}^{\prime} \rightarrow \hat{\Omega}\right) \mathrm{d} \hat{\Omega}^{\prime}=1
$$

Equation (1) indicates that the extinction, absorption, and scattering coefficients together with the scattering phase function and the single scattering albedo are major parameters of the radiation transfer. Thus, the following subsections of the paper are devoted to characterization of the continuous phase layer with dispersed gas bubbles and to the development of detailed models for its effective radiation characteristics.

Let $m_{\lambda}^{\mathrm{d}}=n_{\lambda}^{\mathrm{d}}-i k_{\lambda}^{\mathrm{d}}$ and $m_{\lambda}^{\mathrm{c}}=n_{\lambda}^{\mathrm{c}}-i k_{\lambda}^{\mathrm{c}}$ be the spectral complex indices of refraction of the dispersed phase (i.e., gas bubbles), and of the continuous phase, respectively. The following sections present the formulations for predicting the spectral radiation characteristics of glass containing monodispersed and polydispersed bubbles.

\section{(1) Prediction of Spectral Radiation Characteristics for Monodispersed Bubbles}

We assume here that all the bubbles entrapped in the glass melt have a uniform radius $a$. Then, the effective extinction coefficients (due to absorption and scattering) and the scattering phase function for the layer can be expressed as ${ }^{7}$

$$
\begin{aligned}
\kappa_{\lambda} & =\pi Q_{\mathrm{abs}}^{\mathrm{d}}(a) a^{2} N_{\mathrm{T}}+\left[\kappa_{\lambda}^{\mathrm{c}}-\pi Q_{\mathrm{abs}}^{\mathrm{c}}(a) a^{2} N_{\mathrm{T}}\right] \\
& =\kappa_{\lambda}^{\mathrm{c}}-\pi\left[Q_{\mathrm{abs}}^{\mathrm{c}}(a)-Q_{\mathrm{abs}}^{\mathrm{d}}(a)\right] a^{2} N_{\mathrm{T}} \\
\sigma_{\lambda} & =\pi Q_{\mathrm{sca}}^{\mathrm{d}}(a) a^{2} N_{\mathrm{T}} \\
\beta_{\lambda} & =\left(\kappa_{\lambda}+\sigma_{\lambda}\right) \\
& =\kappa_{\lambda}^{\mathrm{c}}-\pi\left[Q_{\mathrm{abs}}^{\mathrm{c}}(a)-Q_{\mathrm{ext}}^{\mathrm{d}}(a)\right] a^{2} N_{\mathrm{T}}
\end{aligned}
$$

where $Q_{\mathrm{abs}}(a), Q_{\mathrm{sca}}(a)$, and $Q_{\text {ext }}(a)$ denote the absorption, scattering, and extinction efficiency factors and scattering phase function, respectively, for a sphere of radius $a$, while the superscripts " $\mathrm{d}$ " and "c" refer to the dispersed and the continuous phase, respectively. $N_{\mathrm{T}}$ is the total number of bubbles per unit volume, which can be expressed as a function of the void fraction $f_{\mathrm{v}}$ and of the foam and continuous densities, $\rho_{\mathrm{f}}$ and $\rho_{\mathrm{c}}$, respectively:

$$
N_{\mathrm{T}}=\frac{3 f_{\mathrm{v}}}{4 \pi a^{3}}=\frac{3}{4 \pi a^{3}}\left(1-\frac{\rho_{\mathrm{f}}}{\rho_{\mathrm{c}}}\right)
$$

Moreover, for independent scattering, the phase function in a cloud of uniform bubbles $\Phi_{\lambda}(\Theta)$ is the same as that for a single particle $\phi(a, \Theta) ; ;^{10}$ it is also the same for a bubble cloud, i.e.,

$$
\Phi_{\lambda}(\Theta)=\phi(a, \Theta)
$$

Note that the absorption coefficient of the continuous phase $\kappa_{\lambda}^{\mathrm{c}}$ in Eq. (5) can be calculated from the imaginary part $\left(k_{\lambda}^{\mathrm{c}}\right)$ of the complex index of refraction $\left(m_{\lambda}^{\mathrm{c}}\right)$ as

$$
\kappa_{\lambda}^{\mathrm{c}}=4 \pi \eta_{0} k_{\lambda}^{\mathrm{c}}
$$

where $\eta_{0}=v / c_{0}=1 /\left(n_{\lambda}^{\mathrm{c}} \lambda\right)$ is the wavenumber of the wave with a frequency $v$ and phase velocity equal to the speed of light in a vacuum, $c_{0}$.

\section{(2) Spectral Radiation Characteristics for Polydispersed Bubbles}

Figure 1 shows a schematic diagram of layer of continuous condensed phase containing bubbles of different sizes. In glass melt these bubbles may be generated during the batch fusion and fining reactions. ${ }^{3}$ Suppose that all bubbles are spherical (in the case of distorted bubbles one can define an equivalent sphere which preserves the gas volume fraction) and their size (radius $a$ ) distribution is given by the so-called modified gamma function (Ref. 10, pp. 393-94):

$$
n(a)=A a^{\gamma} \exp \left(-B a^{\delta}\right) \quad(0 \leq a<\infty)
$$

The distribution function vanishes at $a=0$ and $a \rightarrow \infty$ and it reaches its maximum at the bubble radius $a_{\max }=(\gamma / \delta B)^{1 / \delta}$. The four constants $A, B, \gamma$, and $\delta$ are taken to be positive and real, and they must be determined from measurable quantities such as the total number of bubbles per unit volume,

$$
\begin{aligned}
N_{\mathrm{T}} & =\int_{0}^{\infty} n(a) \mathrm{d} a \\
& =A \int_{0}^{\infty} a^{\gamma} \exp \left(-B a^{\delta}\right) \mathrm{d} a \\
& =\frac{A \Gamma\left(\frac{\gamma+1}{\delta}\right)}{\delta B^{(\gamma+1) / \delta}}
\end{aligned}
$$

and the bubble density (i.e., the total volume of bubbles per unit volume or the bubble volume fraction) is given by

$$
\begin{aligned}
f_{v} & =\int_{0}^{\infty}\left(\frac{4}{3}\right) \pi a^{3} n(a) \mathrm{d} a \\
& =\frac{4 \pi A \Gamma\left(\frac{\gamma+4}{\delta}\right)}{3 \delta B^{(\gamma+4) / \delta}}
\end{aligned}
$$

Here, $\Gamma(z)$ is the gamma function $\left(=\int_{0}^{\infty} e^{-t} t^{z^{-1}} \mathrm{~d} t\right)$, and it is tabulated by Abramowitz and Stegun. ${ }^{1}$ Note also that the constants $\gamma$ and $\delta$ are usually chosen to be integers.

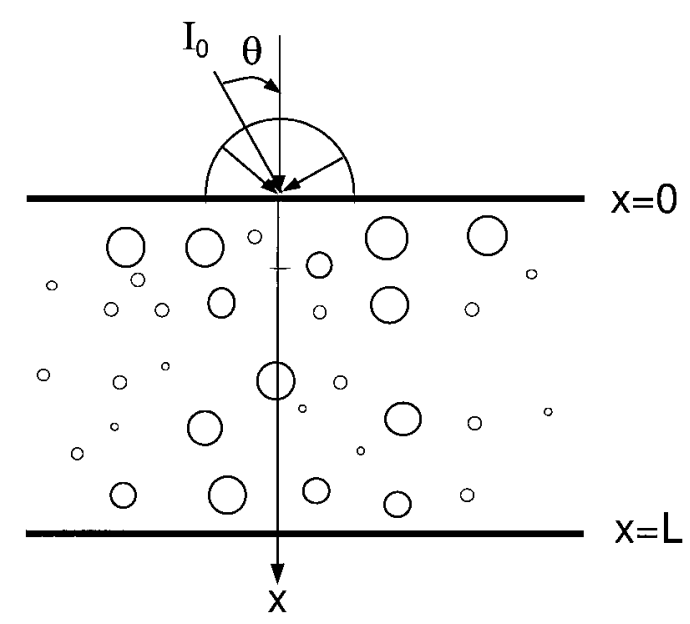

Fig. 1. Schematic of the idealized liquid layer containing bubbles and the coordinate system. 
In the present case, we also assume that all bubbles have the same optical properties throughout the layer. This assumption is valid even if the gas composition in the bubbles changes as the bubbles rise and gases diffuse in and out of the bubbles. Indeed, the absorption of radiation by the gas phase is proportional to the bubble pressure and size whereas scattering depends mainly on the interfacial area. The pressure inside large bubbles is relatively small while that for small bubbles is large but the optical path is small. Thus, whether the bubbles are small or large, the gases contained in the bubbles have little effect on the transfer of radiation. Furthermore, the bubble size distribution function $n(a)$ is assumed to be known; future work on predicting the density function of bubbles transported by the glassmelt convective currents and growing and shrinking due to gas diffusion will be reported soon. Then, the effective extinction coefficients (due to absorption and scattering) and the scattering phase function for the medium containing bubbles can be expressed as ${ }^{7}$

$$
\begin{aligned}
& \kappa_{\lambda}=\pi \int_{0}^{\infty} Q_{\mathrm{abs}}^{\mathrm{d}}(a) a^{2} n(a) \mathrm{d} a+\left[\kappa_{\lambda}^{\mathrm{c}}-\pi \int_{0}^{\infty} Q_{\mathrm{abs}}^{\mathrm{c}}(a) a^{2} n(a) \mathrm{d} a\right] \\
& =\kappa_{\lambda}^{\mathrm{c}}-\pi \int_{0}^{\infty}\left[Q_{\mathrm{abs}}^{\mathrm{c}}(a)-Q_{\mathrm{abs}}^{\mathrm{d}}(a)\right] a^{2} n(a) \mathrm{d} a \\
& \sigma_{\lambda}=\pi \int_{0}^{\infty} Q_{\mathrm{sca}}^{\mathrm{d}}(a) a^{2} n(a) \mathrm{d} a \\
& \beta_{\lambda}=\left(\kappa_{\lambda}+\sigma_{\lambda}\right) \\
& =\kappa_{\lambda}^{\mathrm{c}}-\pi \int_{0}^{\infty}\left[Q_{\mathrm{abs}}^{\mathrm{c}}(a)-Q_{\mathrm{ext}}^{\mathrm{d}}(a)\right] a^{2} n(a) \mathrm{d} a \\
& \Phi_{\lambda}(\Theta)=\frac{\pi}{\sigma_{\lambda}} \int_{0}^{\infty} Q_{\text {sca }}^{\mathrm{d}}(a) \phi(a, \Theta) a^{2} n(a) \mathrm{d} a
\end{aligned}
$$

In calculating the spectral coefficients, most of the computer time is used in evaluating the efficiency factors $Q_{\text {abs }}, Q_{\text {sca }}$, and $Q_{\text {ext }}$ from the Mie theory. In multidimensional and spectral radiative transfer analysis this type of approach becomes impractical. Therefore, it is desirable to have simple approximations for the efficiency factors. The changes in the scattering pattern due to changes in the bubble size should be accounted for in the prediction of the radiation characteristics of the layer containing bubbles. Figure 2 shows the different limiting cases in the $\rho-\chi$

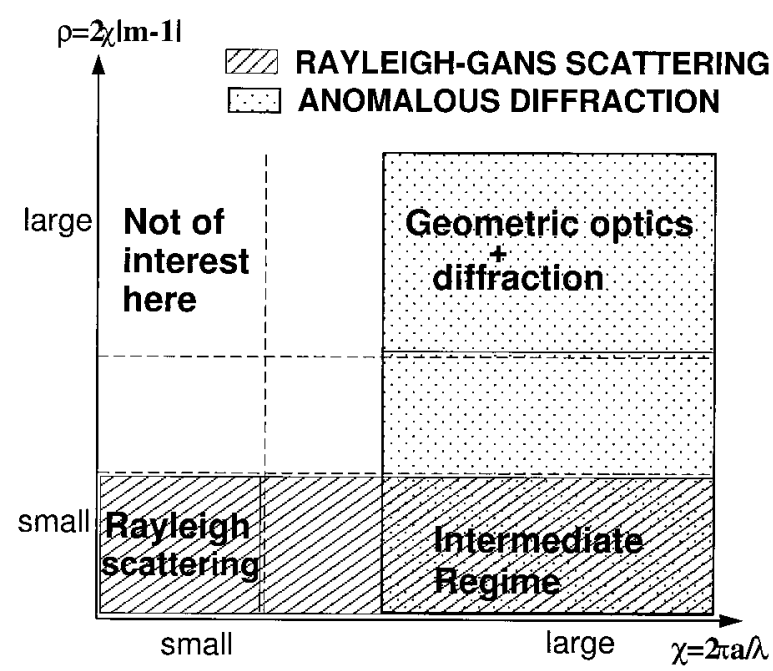

Fig. 2. Survey of the limiting cases in the $\rho-\chi$ domain. domain where simple analytical expressions for $Q_{\mathrm{abs}}(a), Q_{\mathrm{sca}}(a)$, $Q_{\text {ext }}(a)$, and $\phi(a, \Theta)$ are available in the literature. For spheres with index of refraction close to 1 , the $\rho-\chi$ domain can be divided into two limiting cases: ${ }^{13}$

(i) The Rayleigh-Gans scattering domain corresponds to a near-dielectric sphere with (1) $k \approx 0,(2)$ a refractive index of refraction close to unity i.e., $|m-1|<<1$, and such that (3) the phase lag suffered by the central ray that passes through the sphere along a full diameter is small, i.e., $\rho=2 \chi|m-1|<<1$. Then, reflectivity is negligible and the radiation passes through the sphere unattenuated and unrefracted. ${ }^{13}$ The Rayleigh-Gans scattering domain can itself be divided into two limiting cases namely, $\chi \rightarrow 0$ (Rayleigh scattering) and $\chi \rightarrow \infty$ ("intermediate regime").

(ii) The anomalous-diffraction domain is characterized by $\chi \rightarrow \infty$ and $m \rightarrow 1$ corresponding to a straight transmission and subsequent diffraction according to Huygens' principle. ${ }^{13}$ The anomalous-diffraction domain can also be divided into two limiting cases, namely, $\rho \rightarrow 0$ ("intermediate regime") and $\rho \rightarrow \infty$ ("geometrical optics + diffraction regime").

Note that the Rayleigh-Gans domain and the anomalous diffraction domain overlap in the so-called "intermediate regime."

The present work is concerned with relatively large bubbles and wavelengths between 0.4 and $10 \mu \mathrm{m}$ for which the anomalous diffraction is valid. In this region of the $\rho-\chi$ domain, the large gas bubbles are relatively weak absorbers of radiation and mostly act as the strong radiation scatterers. In this case, the approximate analytical expressions for extinction (absorption and scattering) efficiency factors for a weakly absorbing sphere of arbitrary size can be used as derived by van de Hulst (see Ref. 13, p. 179),

$$
\begin{aligned}
& Q_{\text {ext }}\left(\rho^{\prime}, m\right)=2-4\left(\frac{\cos (g)}{\rho^{\prime}}\right)\left[e^{-\rho^{\prime} \tan (g)} \sin \left(\rho^{\prime}-g\right)\right] \\
& +4\left(\frac{\cos (g)}{\rho^{\prime}}\right)^{2}\left[\cos (2 g)-e^{-\rho^{\prime} \tan (g)} \cos \left(\rho^{\prime}-2 g\right)\right]
\end{aligned}
$$

where $\rho^{\prime}=2(n-1) \chi=2(n-1)(2 \pi a / \lambda)$ and $g=\arctan [k /(n-$ $1)]$ are the van de Hulst's normalized size and absorption parameters, respectively, such that $\rho^{\prime d} \tan (g)$ gives the energy absorbed along the axial ray within the sphere. Because of the assumptions of the van de Hulst's theory, the expression overestimates the extinction factor for small spheres and underestimates it for larger spheres. To correct this, Deirmendjian ${ }^{5}$ proposed to use a correction factor $\left(1+D_{i}\right)$. The approach was remarkably successful in improving accuracy of extinction coefficient defined by Eq. (18), and the specific expressions for $D_{i}$ can be found in Ref. 5 (pp. 29-30). The absorption and scattering contributions to the extinction efficiency factor of a single gas bubble are given by the following asymptotic formulas (Ref. 5, p. 35):

$$
\begin{aligned}
& Q_{\text {abs }}\left(\rho^{\prime}, m\right)=1+\frac{e^{-2 \rho^{\prime} \tan (g)}}{\rho^{\prime} \tan (g)}+\frac{e^{-2 \rho^{\prime} \tan (g)}-1}{2\left[\rho^{\prime} \tan (g)\right]^{2}} \\
& Q_{\text {sca }}\left(\rho^{\prime}, m\right)=Q_{\text {ext }}\left(\rho^{\prime}, m\right)-Q_{\text {abs }}\left(\rho^{\prime}, m\right)
\end{aligned}
$$

respectively.

Comparison of the absorption and scattering efficiencies generated using the approximate expressions given by Eqs. (19) and (20) with numerical results obtained from the exact Mie theory (Ref. 5, pp. 30-32) have demonstrated the power of this approach, especially when the precise directional scattering pattern and polarizing properties are not desired. Specifically, the results obtained via the corrected van de Hulst's formula $\left(1+D_{i}\right) Q_{\text {ext }}$ are accurate within about $\pm 0.05 Q_{\text {ext }}$ for a wide range of sphere sizes and indices of refraction. Note that the magnitude of the error increases with an increase in a value of the real part $\left(n_{\lambda}^{\mathrm{d}}\right)$ of the complex index of refraction for both absorbing and nonabsorbing spheres (Ref. 5, p. 32). Of course, if the complex index of refraction of the continuous phase $\left(m_{\lambda}^{\mathrm{c}}\right)$ lies within accuracy limits of the van de Hulst's theory, then Eq. (19) can be successfully used for calculation of the absorption efficiency factor $Q_{\mathrm{abs}}^{\mathrm{c}}(a)$ of the sphere made of the continuous phase as well. 


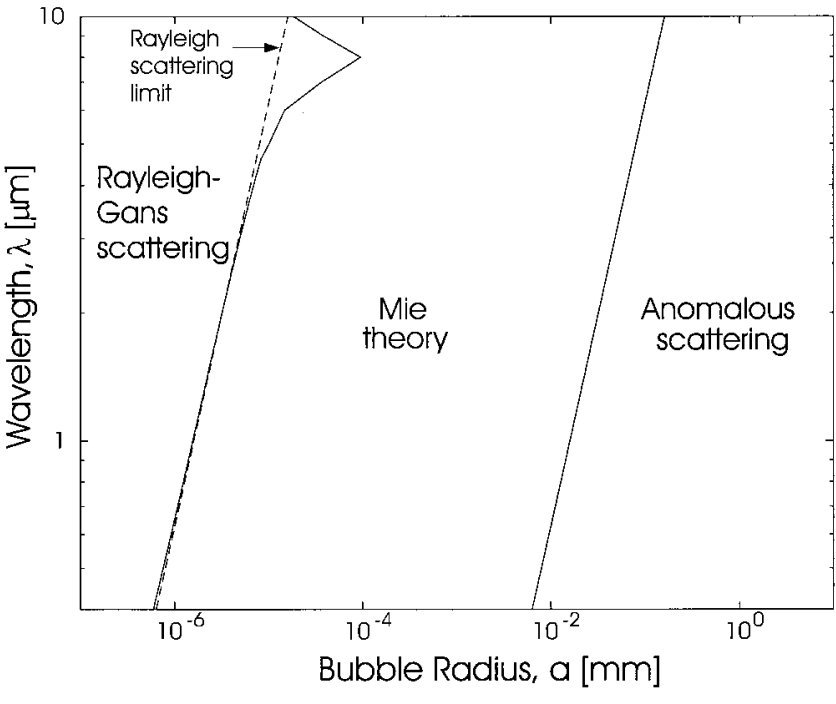

Fig. 3. Map of the scattering theories and approximations used for determining the extinction efficiency factors of the soda-lime silicate containing gas bubbles, i.e., $Q_{\mathrm{abs}}, Q_{\text {sca }}$, and $Q_{\text {ext }}$ in Eqs (5) to (7).

\section{Results and Discussion}

The input parameters for the model include the spectral index of refraction and the spectral absorption coefficient of both the continuous and dispersed phases along with the bubble size distribution and the total number of bubbles per unit volume $N_{\mathrm{T}}$ or the void fraction $f_{\mathrm{v}}$. Clear soda-lime silicate glass (window glass) is used for the sample calculations, and the spectral variation of the real $\left(n_{\lambda}^{\mathrm{c}}\right)$ and imaginary part $\left(k_{\lambda}^{\mathrm{c}}\right)$ of its refractive index $\left(m_{\lambda}^{\mathrm{c}}=n_{\lambda}^{\mathrm{c}}\right.$ $\left.-i k_{\lambda}^{\mathrm{c}}\right)$ are taken from the literature. ${ }^{11}$ The gas mixture contained inside the gas bubbles is transparent to the incident radiation and its complex index of refraction $\left(m_{\lambda}^{\mathrm{d}}\right)$ is assumed to be independent of the wavelength and equal to $1.003-i \cdot 1.0 \times 10^{-10}$. In this section we review the different scattering domains for which simple analytical expressions for the extinction efficiency factors exist. Then, the absorption and extinction coefficients as well as the single scattering albedo of soda-lime silicate glass containing monodispersed and polydispersed bubbles with different size distribution and different void fraction are calculated in the case of anomalous diffraction (large bubbles). Finally, results giving the apparent reflectance, transmittance and absorptance of glass layers of different thickness are discussed.

\section{(1) Model Validity for Glass Containing Bubbles}

(A) Scattering Domains: In defining the limiting cases of the Mie theory for which simple analytical solutions are known, we used the qualitative criteria $\rho<<1$ for Rayleigh-Gans scattering and $\chi>>1$ for anomalous diffraction. For our particular application the different scattering regimes are delimited arbitrarily as follows:

(i) Anomalous scattering approximation is assumed to be valid for $\chi \geq 100$. This condition leads to

$$
a \geq \frac{100 \lambda}{2 \pi}
$$

(ii) The Rayleigh-Gans scattering approximation is assumed to be valid for $\rho \leq 0.01$. For the gas bubbles, this condition is equivalent to

$$
a \leq \frac{\lambda}{400 \pi \sqrt{\left(n_{\lambda}-1\right)^{2}+\left(k_{\lambda}\right)^{2}}}
$$

Note that unlike the index of refraction of the gas contained in the bubbles, that of the soda-lime silicate glass depends on the wavelength and must be accounted for in defining the scattering

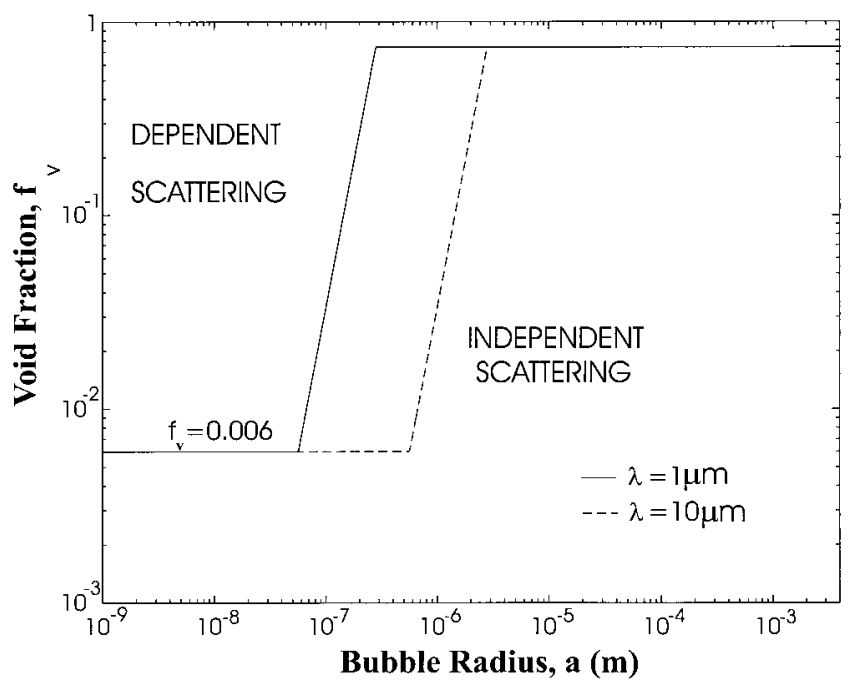

Fig. 4. Scattering regime map for independent and dependent scattering due to spherical bubbles.

domains. The Rayleigh-Gans scattering approximation can be made when the condition expressed by Eq. (22) is valid for both the dispersed and continuous phase.

(iii) A subdomain of the Rayleigh-Gans scattering approximation is the Rayleigh scattering assumed to be valid when $\rho \leq$ 0.01 and $\chi \leq 0.01$. For the gas bubbles and glass spheres, these conditions are expressed by Eq. (22) and

$$
a \leq \frac{\lambda}{200 \pi}
$$

According to Eqs. (5) to (7) one needs to consider the absorption efficiency factor for both gas bubbles and the corresponding glass spheres, and the scattering efficiency factor and the scattering phase function for the gas bubbles in order to predict the effective radiation characteristics of the glass slab containing bubbles. Figure 3 indicates the theory or limiting cases to be used to predict the extinction efficiency factors for both the glass spheres and the gas bubbles in the wavelength-radius domain.

The above representation for the effective radiation characteristics is valid only if the scattering by the ensemble of bubbles is independent, i.e., scattering by one particle is not affected by the presence of surrounding particles. Tien and Drolen ${ }^{12}$ presented a scattering regime map which uses the size parameter $(\chi=2 \pi a / \lambda)$ and the volume fraction $\left(f_{\mathrm{v}}\right)$ as the coordinate axis. They showed that the dependent scattering effects may be ignored as long as $f_{\mathrm{v}}$ $<0.006$ or $c / \lambda<0.5$. Assuming a cubic lattice of bubbles of spacing $p$, the condition $c / \lambda<0.5$ can be expressed in terms of the void fraction as $f_{\mathrm{v}}<(32 \pi / 3)(a / \lambda)=16 \chi / 3$. Figure 4 shows the maximum void fraction $f_{\mathrm{v}}$ for independent scattering as a function of the bubble radius $a$. It suggests that for bubbles larger than $1 \mu \mathrm{m}$ in diameter, independent scattering can be safely assumed.

From Figs. 3 and 4, one can conclude that for bubbles with $a \leq$ $0.1 \mathrm{~mm}$, the radiation characteristics of the glass layer can be predicted from the anomalous diffraction theory. For bubbles less than $1 \mathrm{~nm}$ in diameter, the same radiation characteristics of the

Table I. Major Characteristics of the Uniform Bubble Size Distributions

\begin{tabular}{lcccc}
\hline & & \multicolumn{3}{c}{$N_{\mathrm{T}}\left(\mathrm{no} . / \mathrm{cm}^{3}\right)$} \\
\cline { 3 - 5 } Distribution & $a(\mathrm{~mm})$ & $f_{\mathrm{v}}=0.2$ & $f_{\mathrm{v}}=0.4$ & $f_{\mathrm{v}}=0.6$ \\
\hline Uniform 1 & 0.2 & $5.97 \times 10^{3}$ & $1.19 \times 10^{4}$ & $1.79 \times 10^{4}$ \\
Uniform 2 & 0.8 & 93.3 & 186.5 & 279.8 \\
Uniform 3 & 1.6 & 11.7 & 23.3 & 35.0 \\
\hline
\end{tabular}



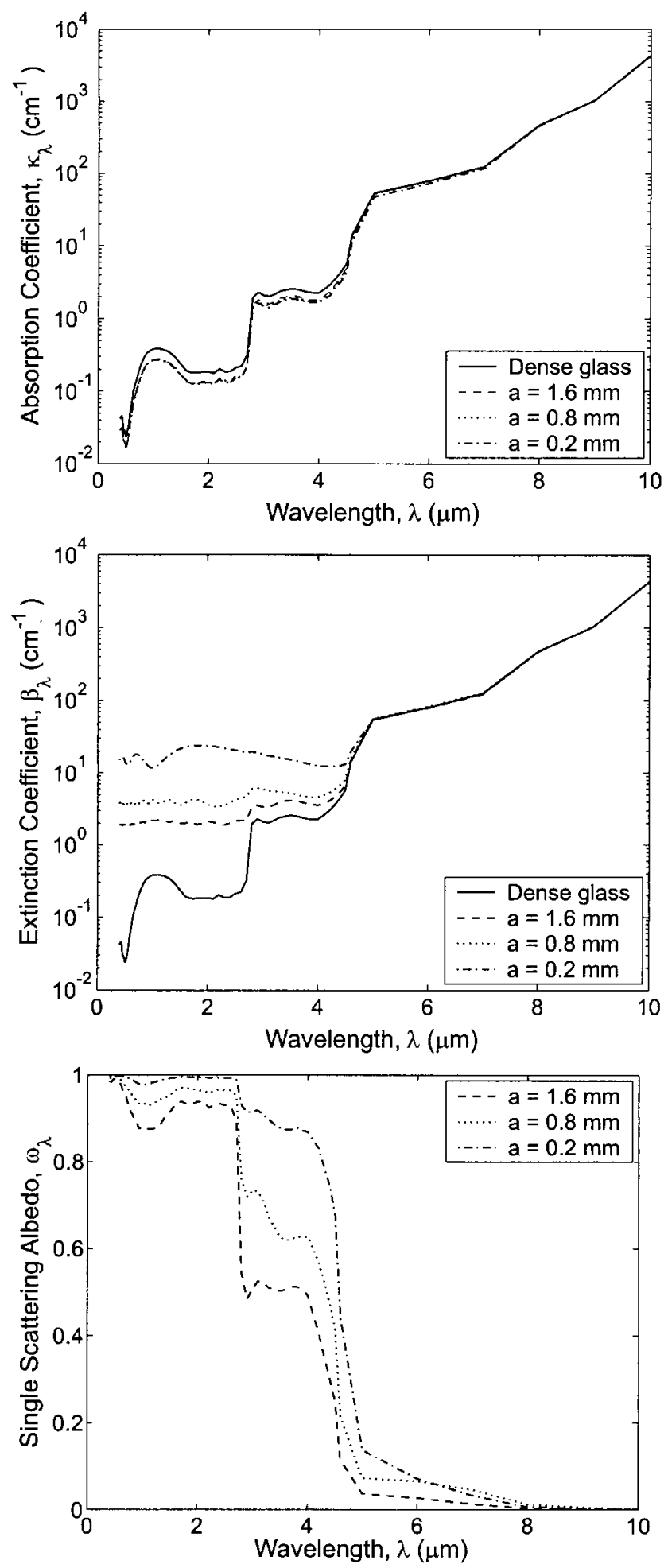

Fig. 5. Effect of bubble radius on the spectral absorption, extinction coefficients, and single scattering albedo for soda-lime silicate glass with $f_{\mathrm{v}}=0.2$.

glass layer can be estimated from the Rayleigh-Gans scattering theory. However, for bubbles having radii between $1 \mathrm{~nm}$ and 0.1 $\mathrm{mm}$ and/or if the void fraction is larger than 0.006, the use of the Mie theory and/or the consideration of dependent scattering is required for wavelengths between 0.4 and $10 \mu \mathrm{m}$, making the computation of the apparent absorptance, reflectance, and transmittance of the layer considerably more involved. Unfortunately, undertaking the task of solving the Mie theory and/or accounting for dependent scattering is beyond the scope of this work.
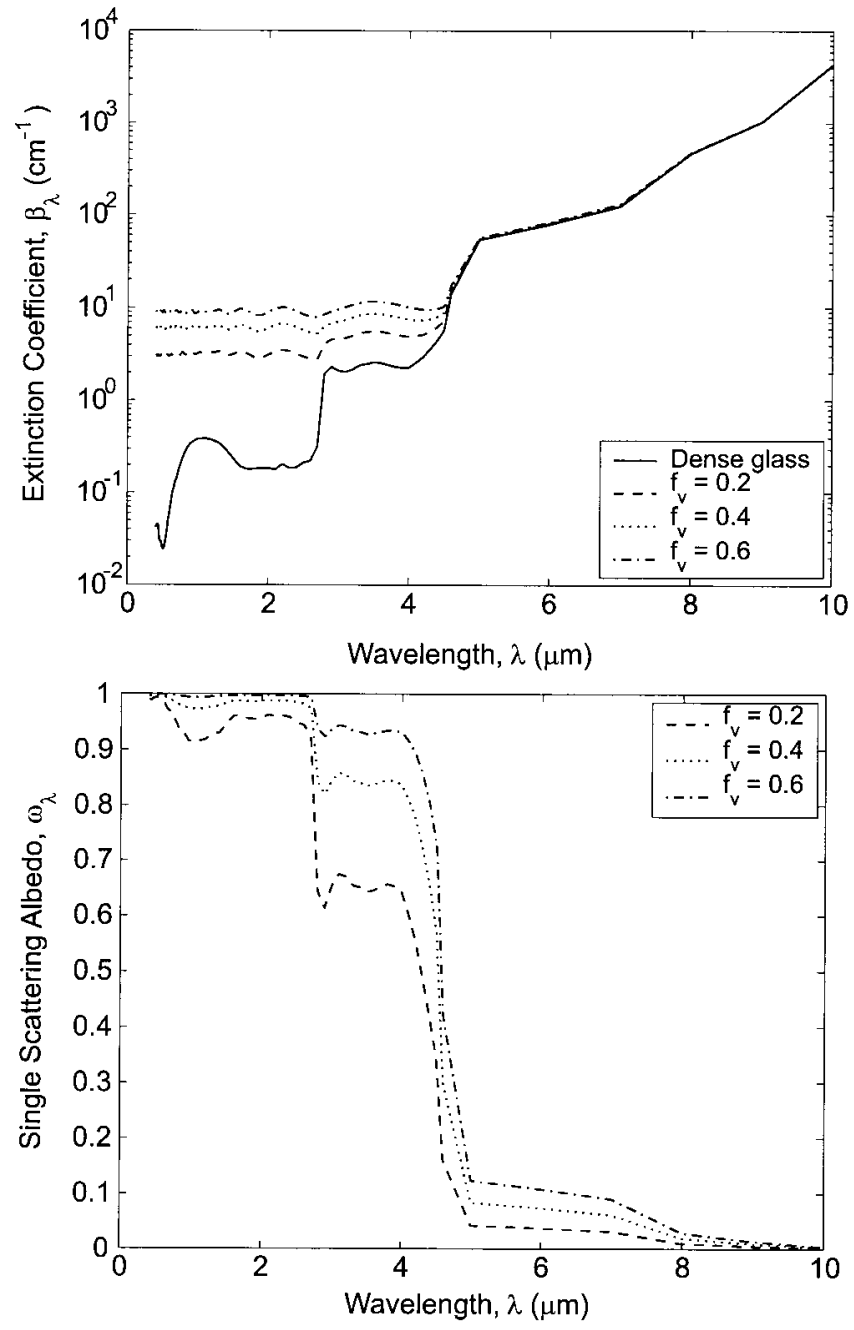

Fig. 6. Effect of void fraction on the spectral extinction coefficient and single scattering albedo for soda-lime silicate glass containing uniform size bubbles $1 \mathrm{~mm}$ in diameter.

Therefore, only large bubbles for which the anomalous diffraction theory and the independent scattering assumption are valid will be considered further.

(B) Ranges of Interest: It can be shown ${ }^{8}$ that bubbles are spherical if their radius $a$ is small compared to the capillary length $l_{\mathrm{c}}\left(a<<l_{\mathrm{c}}\right)$ where the capillary length for gas bubbles surrounded by liquid is defined as

$$
l_{\mathrm{c}}=\sqrt{\frac{2 \gamma}{\left(\rho^{\mathrm{c}}-\rho^{\mathrm{d}}\right) g}}
$$

Here, $\gamma$ is the surface tension $(=300 \mathrm{mN} / \mathrm{m})$, and $\rho^{\mathrm{c}}\left(=2350 \mathrm{~kg} / \mathrm{m}^{3}\right.$ at around $\left.1400 \mathrm{~K}^{9}\right)$ and $\rho^{\mathrm{d}}\left(=1.2 \mathrm{~kg} / \mathrm{m}^{3}\right)$ are the densities of the molten glass and the air, respectively. For soda-lime silicate glass the capillary length is about $4 \mathrm{~mm}$. We assumed that bubbles are spherical for bubble radii up to the capillary length $l_{\mathrm{c}} / 4=1 \mathrm{~mm}$.

The spectral region where the thermal radiation is the most important is considered. The wavelength interval from 0.4 to 10 $\mu \mathrm{m}$ is chosen since it covers nearly $88 \%$ of the thermal radiation emitted by a blackbody at the source temperature of $5800 \mathrm{~K}$ and $94.5 \%$ at $1200 \mathrm{~K}$. In summary, our study is restricted to the size parameters $(\chi=2 \pi a / \lambda)$ ranging from $\chi_{\min }=0.0$ to $\chi_{\max }=$ $3.15 \times 10^{6}$ and a phase shift, $\rho=2 \chi|m-1|$, that can take values from $0(|m-1|<<1$ and $\chi<<1)$ to infinity $(|m-1|<<1$ and $\chi \rightarrow \infty)$. The volume void fraction $f_{\mathrm{v}}$ defined as the ratio of the volume of gas to the total volume can vary between 0 and 0.74 corresponding to the maximum packing of spheres of uniform size, provided that the assumption of dependent scattering is valid. 
Table II. Parameters and Major Characteristics of the Bubble Size Distribution Functions

\begin{tabular}{|c|c|c|c|c|c|c|c|c|}
\hline \multirow[b]{2}{*}{ Distribution } & \multirow[b]{2}{*}{$\gamma$} & \multirow[b]{2}{*}{$\delta$} & \multirow[b]{2}{*}{ A } & \multirow[b]{2}{*}{$B$} & \multicolumn{2}{|c|}{$f_{\mathrm{v}}=0.2$} & \multicolumn{2}{|c|}{$f_{\mathrm{v}}=0.6$} \\
\hline & & & & & $\overline{a_{\max }(\mathrm{mm})}$ & $N_{\mathrm{T}}\left(\mathrm{no} . / \mathrm{cm}^{3}\right)$ & $\overline{a_{\max }(\mathrm{mm})}$ & $N_{\mathrm{T}}\left(\mathrm{no} . / \mathrm{cm}^{3}\right)$ \\
\hline Modified gamma 1 & 4 & 1 & $1.59 \times 10^{10}$ & 80 & 0.5 & 116.4 & 0.5 & 349.2 \\
\hline Modified gamma 3 & 8 & 1 & $8.22 \times 10^{13}$ & 80 & 1.0 & 24.7 & 1.0 & 74.1 \\
\hline
\end{tabular}

\section{(2) Radiation Characteristics of the Glass}

\section{Containing Bubbles}

In this section we first discuss the effect of the void fraction and of the bubble radius on the radiation characteristics of soda-lime silicate glass containing bubbles of uniform size. Then, polydispersed bubble clouds are considered and the results on the effect of their size distribution are presented.

(A) Uniform Distribution: Effect of Bubble Radius and Void Fraction: As a practical example, the spectral absorption and extinction coefficients as well as the single scattering albedo have been predicted for clear soda-lime silicate glass containing monodispersed bubbles for a volumetric void fraction of 0.2 . Three different radii $a$ were considered-0.2, 0.8 , and $1.6 \mathrm{~mm}$. Note that in the limiting case when $f_{\mathrm{v}}=0$, i.e., for dense glass, the scattering coefficient $\sigma_{\lambda}$ and the single scattering albedo $\omega_{\lambda}$ vanish. Table I summarizes the simulations for monodispersed bubbles and the corresponding number of bubbles contained per cubic centimeter of glass.

From Fig. 5 one can see that the presence of bubbles reduces the absorption coefficient in the spectral region of 0.4 to $4.5 \mu \mathrm{m}$ where the absorption coefficient of the glass is relatively small. In this same region, the extinction coefficient is strongly affected by the presence and the size of the bubbles. The scattering is particularly important for smaller bubbles and the single scattering albedo is close to unity. This indicates that the radiative transfer is dominated by scattering rather than by absorption for $0.4<\lambda<4.5$ $\mu \mathrm{m}$. In the spectral region 4.5 to $10 \mu \mathrm{m}$, however, the absorption coefficient of the dense glass is large and the presence and the size of the bubbles have little effect of the effective absorption coefficient of the glass layer. In other words, the scattering coefficient is negligible compared with the absorption coefficient and the radiative transfer is dominated by absorption.

Moreover, the absorption coefficient decreases significantly as the void fraction increases and can be reduced by up to one order of magnitude for void fractions $f_{\mathrm{v}}$ varying from 0.2 to 0.6 (Fig. 6). In contrast, the extinction coefficient and the single scattering albedo increase as the void fraction or the number of bubbles

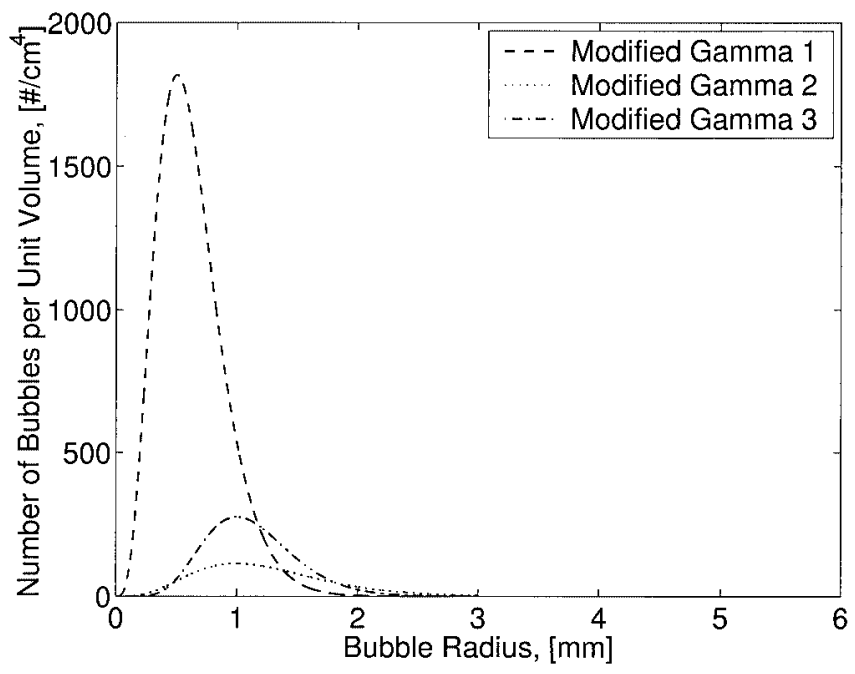

Fig. 7. Typical bubble size distributions as summarized in Table I for $f_{\mathrm{v}}=0.2$.
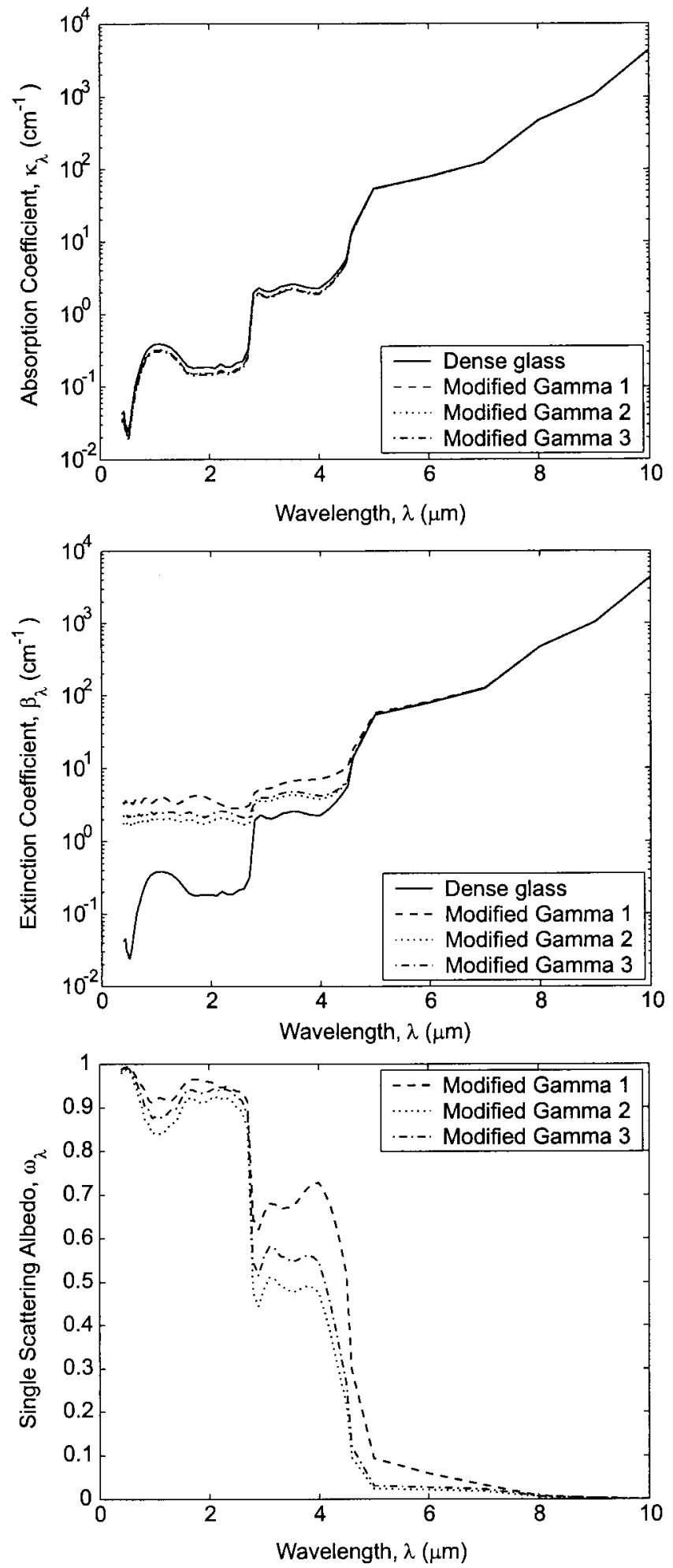

Fig. 8. Effect of size distribution on the spectral absorption, extinction coefficients, and single scattering albedo of soda-lime silicate glass with $f_{\mathrm{v}}=0.2$. 

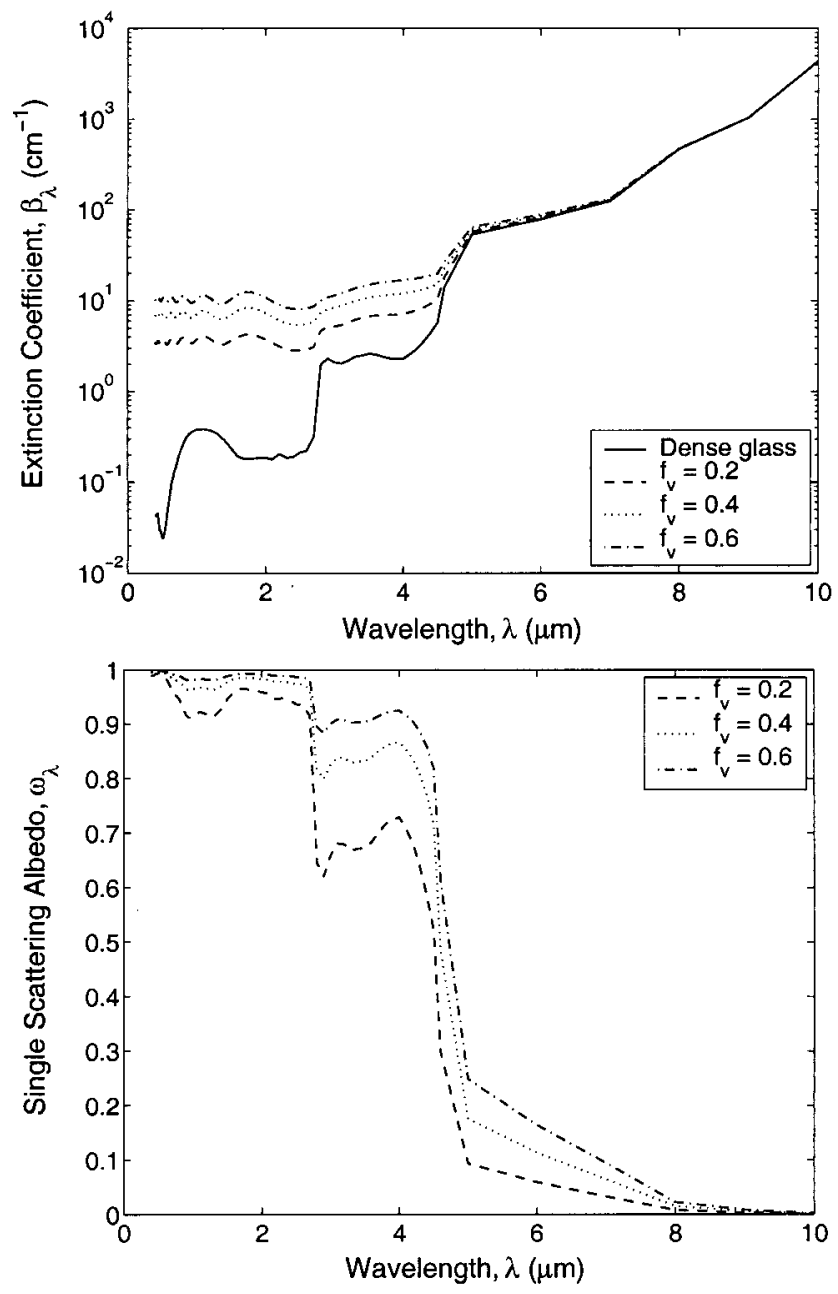

Fig. 9. Effect of void fraction on the spectral extinction coefficient and single scattering albedo for soda-lime silicate glass containing gas bubbles for modified gamma distribution function 1 .

increase. This can be explained by the fact that increasing the void fraction increases the number of scatterers, while the absorption by the two-phase mixture decreases.

(B) Effect of Bubble Size Distribution: So far, the bubble size distribution was assumed to be uniform. However, in reality bubbles entrapped in the glass melt are of different diameters. Sample calculations were performed for the spectral radiation characteristics of glass containing different bubble size distributions but with a constant void fraction. Table II summarizes the conditions simulated while Fig. 7 shows the corresponding bubble density functions. The effect of the maximum radius $a_{\max }$ and of $\gamma$ are assessed while the parameter $\delta$ is taken to be unity (gamma function). Figure 8 shows the absorption and extinction coefficients and the single scattering albedo for different bubble size distributions at a void fraction of 0.2. Figure 9 illustrates the effect of void fraction on the radiation characteristics for the first bubble size distribution. In general, one can see that the bubble size distribution has a strong influence on the extinction coefficient and single scattering albedo of glass containing bubbles but very little on the absorption coefficient. It is interesting to note that the void fraction seems to affect the extinction coefficient significantly for the wavelength range of $0.4 \mu \mathrm{m} \leq \lambda \leq 4.5 \mu \mathrm{m}$. Indeed, increasing in the void fraction from 0.2 to 0.6 leads to an increase of the extinction coefficient by a factor of 3 while the relative effect of the distribution at $f_{\mathrm{v}}=0.6$ is similar to that at $f_{\mathrm{v}}=0.2$.

\section{Concluding Remarks}

This paper has presented an analysis of radiative transfer in a semitransparent glass layer containing gas bubbles with application to glass processing and manufacturing. The results of sample calculations performed lead to the following conclusions:

(1) For gas bubbles smaller than $10 \mu \mathrm{m}$ in diameter and void fractions larger than 0.006 , the Mie theory should be used and/or considerations of dependent scattering are required.

(2) For gas bubbles larger than $0.1 \mathrm{~mm}$ in radius the analysis developed for glass foams by Fedorov and Viskanta ${ }^{6,7}$ can be extended over the entire range of void fractions (from 0 to 0.74 ).

(3) Even small void fractions affect the total apparent radiation characteristics of the glass layer containing large bubbles. The effect of the void fraction is even more significant for thickner layers and where the bubble size distribution is not uniform. Therefore, in modeling the radiative heat transfer in glass melting furnaces one should consider the effects of gas bubbles on the radiation characteristics of the glass melt since bubbles are always present in industrial glass melting furnaces where they exist in large numbers.

(4) The model could be used as a nonintrusive method for measuring void fraction and bubble size distribution in two-phase flows by using infrared spectroscopy and inverse methods in spectral regions where the liquid phase is weakly absorbing.

Finally, the radiation characteristics of fused quartz containing bubbles have been recently determined from experimental measurements of spectral bidirectional transmittance and reflectance using an inverse method. ${ }^{16}$ 


\section{Nomenclature}

a Bubble radius

$c_{0} \quad$ Speed of light under vacuum

$D \quad$ Deirmendjian's correction factor

$F \quad$ Azimuth-averaged scattering phase function

$f_{\mathrm{v}} \quad$ Bubble void volume fraction

$g \quad$ Specific gravity or van de Hulst's absorption parameter, $\arctan \left[k_{\lambda}^{\mathrm{d}} /\left(n_{\lambda}^{\mathrm{d}}-1\right)\right]$

I Radiation intensity

$I_{\mathrm{b}} \quad$ Blackbody radiation intensity

$I_{0} \quad$ Incident intensity of collimated radiation

$Q \quad$ Efficiency factor

$k \quad$ Imaginary part of the complex index of refraction

$l \quad$ Thickness of the slab

$m \quad$ Complex index of refraction, $n-i k$

$N_{\mathrm{T}} \quad$ Total number of bubbles per unit volume

$n \quad$ Real part of the complex index of refraction

$r \quad$ Reflectivity of the interface

$\hat{s} \quad$ Spatial coordinate vector

$x \quad$ Local depth of the slab (Fig. 1)

\section{Greek symbols}

$\chi \quad$ Size parameter, $2 \pi a / \lambda$

$\beta \quad$ Extinction coefficient, Eq. (7)

$\gamma \quad$ Surface tension

$\kappa \quad$ Absorption coefficient, Eq. (5)

$\sigma \quad$ Scattering coefficient, Eq. (6) or Stefan-Boltzmann constant

$\lambda \quad$ Wavelength of the incident radiation

$\eta \quad$ Wavenumber of the incident radiation, $v / c_{0}$

$\nu \quad$ Frequency of the incident radiation

$\hat{\Omega} \quad$ Line-of-sight direction

$\omega \quad$ Single scattering albedo, $\sigma_{\lambda} /\left(\kappa_{\lambda}+\sigma_{\lambda}\right)$

$\Phi \quad$ Effective scattering phase function, Eq. (9)

$\phi \quad$ Scattering phase function of the single bubble

$\varphi \quad$ Azimuth angle

$\rho \quad$ Density or phase shift, $2|m-1| \chi$

$\rho^{\prime} \quad$ van de Hulst's normalized size parameter,

$$
2(n-1) \chi
$$

$\Theta \quad$ Scattering angle

$\tau \quad$ Optical depth, $\int_{0}^{x}\left(\kappa_{\lambda}+\sigma_{\lambda}\right) \mathrm{d} x$

\section{Subscripts}

$\lambda \quad$ Refers to wavelength-dependent quantity

abs Refers to absorption

c Refers to continuous phase

d Refers to dispersed phase

ext Refers to extinction

sca Refers to scattering

\section{Superscripts}

d Refers to dispersed phase (gas bubble)

c Refers to the continuous phase

\section{Acknowledgments}

We are indebted to Dr. A. G. Fedorov for helpful discussions and exchange of information.

\section{References}

${ }^{1}$ M. Abramowitz and I. A. Stegun, Handbook of Mathematical Functions. Dover Publications, New York, 1965

${ }^{2}$ B. Balkanli and A. Ungan, "Numerical Simulation of Bubble Behaviour in Glass Melting Tanks. Part 1. Under Ideal Conditions," Glass Technol., 37 [1] 29-34 (1996).

${ }^{3}$ R. G. C. Beerkens, "The Role of Gases in Glass Melting Processes," Glastech. Ber., 71 [12] 369-80 (1995).

${ }^{4}$ K.-B. Cheong, K.-M Moon, and T.-H. Song, "Treatment of Radiative Transfer in Glass Melts: Validity of Rosseland and P-1 Approximations," Phys. Chem. Glasses, 40 [1] 26-33 (1999).

${ }^{5}$ D. Deirmendjian, Electromagnetic Scattering on Spherical Polydispersions. Elsevier, New York, 1969.

${ }^{6}$ A. G. Fedorov and R. Viskanta, "Radiative Characteristics of Glass Foams," J. Am. Ceram. Soc., 83 [11] 2769-76 (2000)

${ }^{7}$ A. G. Fedorov and R. Viskanta, "Radiative Transfer in a Semitransparent Glass Foam Blanket," Phys. Chem. Glasses, 41 [3] 127-35 (2000).

${ }^{8}$ E. Franses, "Interfacial Phenomena: Principles, Data, and Applications"; Ch. 2. Class Notes ChE 668, Purdue University, West Lafayette, IN, 2000.

${ }^{9}$ P. R. Laimbock, "Foaming of Glass Melts"; Ph.D. Thesis. Technical University of Eindhoven, Eindhoven, Netherlands, 1998

${ }^{10}$ M. F. Modest, Radiative Heat Transfer. McGraw-Hill, New York, 1993.

${ }^{11}$ M. Rubin, "Optical Properties of Soda-Lime Silicate," Sol. Energy Mater. 12, 275-88 (1985)

${ }^{12}$ C. L. Tien and B. L. Drolen, "Thermal Radiation in Particulate Media with Dependent and Independent Scattering"; pp. 1-32 in Annual Review of Numerical Fluid Mechanics and Heat Transfer, Vol. 1. Edited by T. Chawla. Hemisphere, New York, 1987.

${ }^{13}$ H. C. van de Hulst, Light Scattering by Small Particles. Wiley, New York, 1957.

${ }^{14} \mathrm{R}$. Viskanta, "Review of Three-Dimensional Mathematical Modeling of Glass Melting," J. Non-Cryst. Solids, 177, 347-62 (1994).

${ }^{15}$ R. Viskanta and M. P. Menguc, "Radiative Transfer in Combustion System," Prog. Energy Combust. Sci. 13, 97-160 (1987).

${ }^{16}$ D. Baillis, F. Randrianalisoa, L. Pilon, and R. Viskanta, "Identification of Radiative Characteristics of Fused Quartz Containing Bubbles Using Discrete Ordinates Method with Fresnel Interfaces"; pp. 215-24 in Computational Thermal Radiation in Participating Media (EUROTHERM Seminar 73, Mons, Belgium, April 15-17, 2003). 